\title{
Inter- and Intra-Hemispheric Fibers of Olfactory and Motor Areas in Kallmann Syndrome with Defective Corpus Callosum
}

Yuri Masaoka ${ }^{1,2^{*}}$, Andrew Zalesky², Masaru Tatsuno ${ }^{3}$, Shingo Okamoto ${ }^{4}$, Masaki Yoshida $^{5}$, Nobuyoshi Koiwa ${ }^{6}$, Mutsutaka Kobayakawa $^{7}$, Keiko Watanabe ${ }^{1}$, Masahiro Ida ${ }^{8}$, Ikuo Homma ${ }^{9}$, Masahiko Izumizaki ${ }^{1}$ and Christos Pantelis ${ }^{2}$

${ }^{1}$ Department of Physiology, Showa University School of Medicine, Tokyo, Japan

${ }^{2}$ Melbourne Neuropsychiatry Centre, Department of Psychiatry, University of Melbourne and Melbourne Health, Melbourne, VIC, Australia

${ }^{3}$ Department of Pediatrics, Showa University School of Medicine, Tokyo, Japan

${ }^{4}$ Okamoto Children's Clinic, Nara, Japan

${ }^{5}$ Department of Ophthalmology, Jikei Medical University, Tokyo, Japan

${ }^{6}$ Department of Health and Science, University of Human Arts and Sciences, Saitama, Japan

${ }^{7}$ Tokyo University of Information Science, Tokyo, Japan

${ }^{8}$ Department of Radiology, Stroke Center, Ebara Tokyo Hospital

${ }^{9}$ Tokyo Ariake University of Medical and Health Sciences, Tokyo, Japan

\begin{abstract}
Kallmann syndrome (KS) is a form of hypogonadotropic hypogonadism with variable disturbances of olfaction, with some patients having complete anosmia. In this study, we report observations of cerebral white matter connectivity in a rare patient diagnosed with KS associated with a defective corpus callosum (DCC) (KS-DCC) using non-invasive tractography-based diffusion tensor imaging (DTI). In the partial CC, two genua remnants were observed. The KS-DCC patient had a normal life without cognitive impairment and had a normal intelligence quotient, manifesting only the two common symptoms hypogonadotropic hypogonadism and anosmia. Mirror movements were not observed. Compared with normal, DTI tractography of the olfactory related area in the KS-DCC patient revealed fewer inter-hemispheric fibers between right and left olfactory areas (including olfactory tubercle and anterior commissure). Overall, the KSDCC patient had higher numbers of intra-hemispheric fibers bilaterally and fewer inter-hemispheric connecting fibers. The higher numbers of intra-hemispheric fibers connected the right thalamus and right supplementary motor area (SMA) and the left thalamus and left SMA. The fewest number of fibers observed in the KS-DCC patient were those connecting right and left SMA. The Probst bundle together with increased intra-hemispheric fibers may indicate plastic rewiring of corpus callosum connections.
\end{abstract}

Keywords: Defective corpus callosum; Kallmann syndrome; Tractography; Olfaction; Intra- and inter-hemisphere

\section{Introduction}

Kallmann syndrome (KS) is a genetically mediated form of hypogonadotropic hypogonadism that is associated with variable disturbances of olfaction, with some patients having complete anosmia. The prevalence of KS has been estimated at 1:10,000 in males, while in females it is 5-7 times lower [1]. Most patients are diagnosed during adolescence, when an absence of spontaneous puberty is observed simultaneously with impaired olfactory ability.

$\mathrm{KS}$ is genetically heterogeneous with mutations in an X-linked gene, $K A L-1$, located at Xp22.3 [2]. KAL-1 encodes anosmin-1 (680 amino acids) that has a role in neuronal migration and axon path-finding [3]. Loss-of-function mutations in KAL-1 results in agenesis of the olfactory bulb and olfactory tract, and hypogonadotropic hypogonadism because of an arrest of neuronal migration involving hypothalamic neurons for gonadotropin releasing hormone $(\mathrm{GnRH})$ and olfactory neurons in the olfactory placode that have a common embryonic origin $[2,4]$. Disturbances in onset of puberty result, as GnRH is a key regulatory molecule for reproduction and sexual behavior. Since anosmin-1 is important for axonal guidance and branching of these neurons, other neurological symptoms are also observed in KS patients, such as mirror movements (involuntary movements of one side of the body that accompany and mirror intentional movements of the other side), hearing loss, abnormal visual attention, and oculomotor abnormalities are observed in KS patients [3].

In this study, we report observations of cerebral white matter connectivity in KS associated with a defective corpus callosum (DCC) (KS-DCC), using non-invasive tractography-based diffusion tensor imaging (DTI) [5]. This KS-DCC patient had only two common symptoms related to KS, namely, hypogonadotropic hypogonadism and anosmia; he had an otherwise normal life without apparent cognitive impairment and no evidence of other symptoms. Importantly, there was a notable absence of mirror movements.

Our aim was to examine white-matter connectivity in this patient using tractographic methods [6]. This was achieved by examining nodes within a network, with each node encapsulating a distinct graymatter region; pairs of nodes were linked if they were interconnected via an axonal fiber [7]. Based on the patient's symptoms of anosmia and the absence of mirror movements, we investigated cortico-cortical connectivity in this patient compared with healthy control subjects, focusing on the following areas: 1) olfactory afferent fibers from the olfactory tract and their connections with other olfactory related regions including the piriform cortex (Pir), the amygdala (AMG), entorhinal cortex (ENT), hippocampus (HI) and orbitofrontal cortex (OFC); 2) intra- and inter-hemispheric fiber connectivity of motor areas.

*Corresponding author: Yuri Masaoka, 1-5-8, Hatanodai, Shinagawa-ku, Tokyo 142-8555, Japan, Tel: +81 33784 8113; Fax: +81 33784 0200; E-mail: faustus@med.showa-u.ac.jp

Received May 08, 2016; Accepted June 13, 2016 ; Published June 20, 2016

Citation: Masaoka Y, Zalesky A, Tatsuno M, Okamoto S, Yoshida M, et al. (2016) Inter- and Intra-Hemispheric Fibers of Olfactory and Motor Areas in Kallmann Syndrome with Defective Corpus Callosum. J Neurol Neurophysiol 7: 380. doi:10.4172/2155-9562.1000380

Copyright: (c) 2016 Masaoka Y, et al. This is an open-access article distributed under the terms of the Creative Commons Attribution License, which permits unrestricted use, distribution, and reproduction in any medium, provided the original author and source are credited. 
Citation: Masaoka Y, Zalesky A, Tatsuno M, Okamoto S, Yoshida M, et al. (2016) Inter- and Intra-Hemispheric Fibers of Olfactory and Motor Areas in Kallmann Syndrome with Defective Corpus Callosum. J Neurol Neurophysiol 7: 380. doi:10.4172/2155-9562.1000380

\section{Materials and Methods}

\section{Subjects and clinical evaluation}

We evaluated a 19 year old patient with KS-DCC. Data from five age-matched healthy subjects (mean age $19 \pm 0.5$ years old) were used as controls. The patient and controls were all right-handed males according to the Edinburgh Handedness Inventory [8]. The study was approved by the ethics committee of Showa University School of Medicine and all participants provided written informed consent.

The patient was referred from Okamoto Children's Clinic in Nara, Japan with a diagnosis of KS. Criteria for the diagnosis of KS included clinical reports of anosmia and features of hypogonadism. The patient was referred to our unit for further investigation because of lack of onset of puberty and hypogonadism with absent facial hair and sparse pubic hair (Tanner stage 1). The patient had cryptorchidism as an infant, and did not have renal anomalies or cleft lip/palate. His height was $178.7 \mathrm{~cm}$, with weight of $77.2 \mathrm{~kg}$. Absence of the bilateral olfactory bulb $(\mathrm{OB})$ was confirmed by $\mathrm{T} 1$-weighted $3 \mathrm{~T}$ magnetic resonance imaging (MRI) and was determined in a coronal plane covering the anterior and middle segments of the base of the skull according to the definition of a prior study [9]. Patient's hypothalamus and the pituitary were normal on imaging. Laboratory endocrine status showed low testosterone level $(0.09 \mathrm{ng} / \mathrm{ml}$; normal range, $2.6-6.9 \mathrm{ng} / \mathrm{ml})$, and low basal serum luteinizing hormone (LH: 0.1 IU/L; normal range, 2.3-6.6) and follicle stimulating hormone (FSH: $0.4 \mathrm{IU} / \mathrm{L}$; normal range, $2.1-$ 6.6) concentrations. Left and right scrotal volumes were $3 \mathrm{ml}$ (normal for age, 15 to $30 \mathrm{ml}$ ).

Figure 1 shows a sagittal section of the patient's original anatomical MRI (left), with the brain normalized according to the pipeline method (described in the Acquisition and preprocessing of images) (Figure 1: middle) and superimposed on the Montreal Neurological Institute (MNI) standard brain atlas, indicated in red (Figure 1: Right).

In the partial CC, two genua remnants were observed. The center of the anterior remnant was located at MNI coordinates: $\mathrm{x}: 90, \mathrm{y}: 131, \mathrm{z}$ : 86 (normally defined as anterior horn of the lateral ventricle according to Harvard-Oxford Subcortical Structural Atlas) and at the center of the posterior remnant located at: x: 90, y: 100, z: 84 (defined as third ventricle).

The KS-DCC patient had no subjective restrictions in daily life and had been studying at a Japanese university over the previous two years. He had a normal intelligence quotient (IQ); assessed by the Wechsler Adult Intelligence Scale (WAIS) [10], including full-scale IQ (FSIQ), verbal comprehension index, working memory index, perceptual organization index and processing speed index (Table 1). Years of education were similar between the patient and controls (Table 1). The patient had no prior history of psychiatric disorders based on DSM IV criteria, or drug abuse.

Age matched control subjects were recruited from student volunteers of the University of Human Science in Japan (19.6 \pm 0.5$)$. All control subjects had no structural abnormalities or white-matter hyperintensities on MRI. All controls had average levels of IQ as measured by WAIS [10] (Table 1), and had no history of psychiatric disorders or drug abuse.

\begin{tabular}{|l|c|c|c|}
\hline \multirow{2}{*}{ Age } & & Control & Kall-DCC \\
\hline Year of Education & & $19.6 \pm 0.5$ & 19 \\
\hline \multirow{3}{*}{ Taste Identification Test } & Detection Score & $\begin{array}{c}0.56 \pm 0.2 \\
(\text { Normal) }\end{array}$ & Impaired \\
\cline { 2 - 4 } & Recognition Score & $\begin{array}{c}0.16 \pm 0.3 \\
(\text { Normal) }\end{array}$ & Impaired \\
\hline \multirow{2}{*}{ WAIS Full IQ } & Sweet & $2.8 \pm 0.4$ & 3 \\
\hline Verbal Comprehension Index & Salty & $1.8 \pm 0.4$ & 2 \\
\hline Working Memory Index & Sour & $2.8 \pm 0.4$ & 2 \\
\hline Perceptual Organization Index & Bitter & $2.8 \pm 0.4$ & 2 \\
\hline Processing Speed Index & & $100 \pm 12$ & 113 \\
\hline Mirror Movement & & $108 \pm 10$ & 114 \\
\hline
\end{tabular}

Table 1: Summary of the KS-DCC patient and control characteristics.

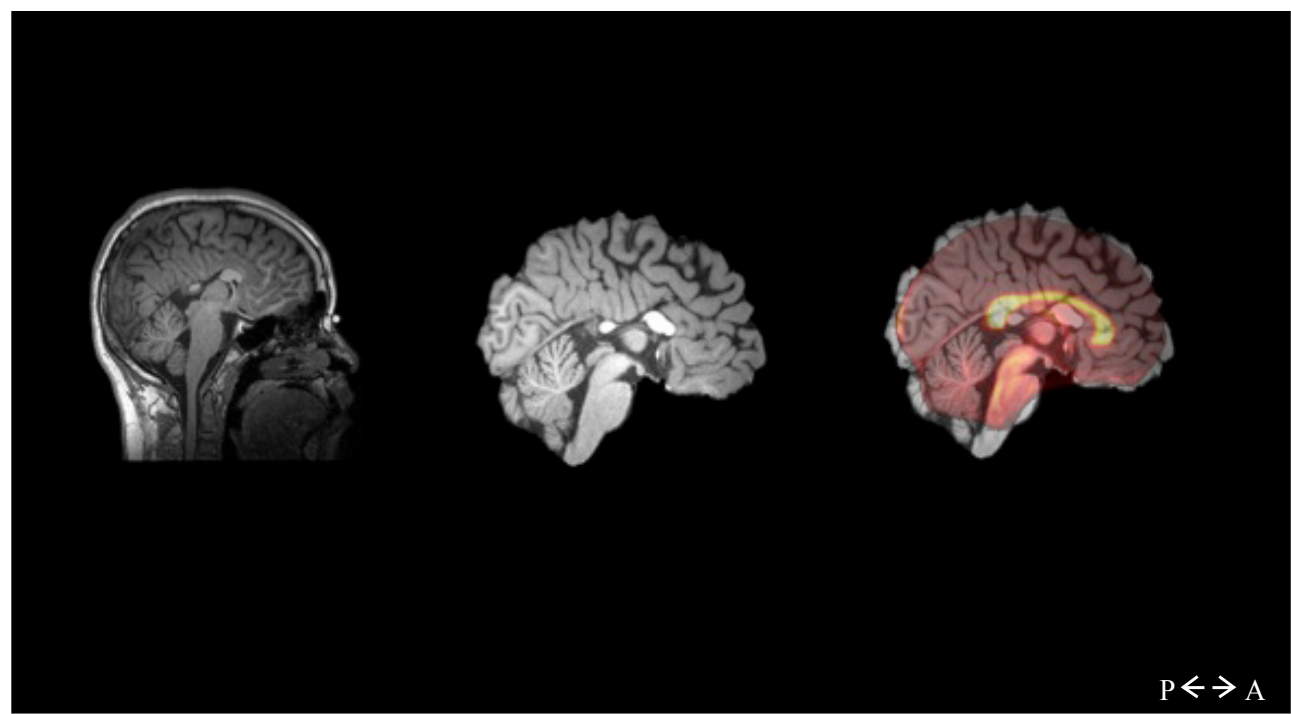

Figure 1: A sagittal section of the patient's original anatomical MRI (left), with the normalized patients' brain (middle) and the normalized patients' brain superimposed on the Montreal Neurological Institute (MNI) standard brain (right), A, Anterior; P, Posterior. 
Olfactory detection and identification acuities were evaluated by the T\&T olfaction test to confirm anosmia in the patient and the normal range in controls. Details of the olfactory acuity test were previously described [11]. In brief, the T\&T olfaction test consists of five odors: A, $\beta$-phenyl ethyl alcohol; $\mathrm{B}$, methyl cyclopentenolone; $\mathrm{C}$, isovaleric acid; D, $\gamma$-undecalactone; and E, skatole. Each odorant was dissolved in propylene glycol and presented at eight different concentrations. In the test for each subject, we applied each odorant starting at the lowest concentration and progressively increasing the concentration. During each test, the subject was asked whether an odor was detected. When the subject perceived an odor, they were required to identify and describe the odor. The concentration at which the odor was perceived, but not identified, was considered the detection level. The concentration at which an odor was first identified was considered the recognition level. Each subject's odor detection threshold was expressed as the mean of all odor threshold scores as follows: $(\mathrm{A}+\mathrm{B}+\mathrm{C}+\mathrm{D}+\mathrm{E}) / 5$.

Taste sensitivities were tested using semiquantitative clinical gustometry using filter-paper discs (filter-paper disc method; Taste disc, Sanwa Chemical Laboratory, Nagoya, Japan). This semi-quantitative method is routinely used for the evaluation of dysgeusia in clinical settings [12]. In brief, recognition thresholds for four basic tastes (sweet, salty, sour and bitter) were evaluated using the same chemical solutions (sucrose, $\mathrm{NaCl}$, tartaric acid and quinine, respectively).

\section{Mirror movements}

Mirror movements were evaluated by a test adapted from Mayston et al. [13]. In brief, subjects held their palms facing up with fingers extended on a table. The subjects sequentially opposed the tip of each finger starting with the thumb then the index and little fingers and then subjects' repeated this in the opposite direction. Subjects were asked to flex each finger in turn several times. Mirror movements were assessed as positive if an involuntary movement of the homologous finger of the other hand was noted. Absence of mirror movements were confirmed by surface electromyogram (EMG) recorded from the first dorsal interosseous muscle of both hands using $\mathrm{Ag}-\mathrm{AgCl}$ electrodes in a belly tendon montage.

\section{Acquisition and preprocessing of images}

Each participant was scanned on a 3T MAGNETOM Trio, a Tim System (Siemens Healthcare, Erlangen, Germany) with a 32-channel head coil, located at Ebara Hospital, Tokyo, Japan. An anatomical MRI scan was acquired using T1-weighted 3D-magnetization-prepared rapid acquisition by gradient echo (MPRAGE) in sagittal orientation. Controls and patient MRI were acquired using the same parameters: $\mathrm{TR}=2300 \mathrm{~ms}$; $\mathrm{TE}=2.98 \mathrm{~ms}$; flip angel=9'; FOV-256 mm; matrix: 256 $\times 256$; section resolution equal to $1.0 \times 1.0 \times 1.0 \mathrm{~mm}$, producing an isometric voxel of $1 \mathrm{~mm}^{3}$. Sixty-four gradient-weighted volumes applying monopolar diffusion scheme were acquired using a spinecho planar imaging (EPI), prototype sequence with the following parameters: $b$-value $1000 \mathrm{~s} / \mathrm{mm}^{2} ; 65$ consecutive axial slices of thickness $1.5 \mathrm{~mm} ; 140 \times 140$ image matrix with an in-plane voxel resolution of 1.5 $\times 1.5 \mathrm{~mm}^{2}$; field of view $189 \times 189 \mathrm{~mm}^{2}$; TR=8.84 s; TE=88 ms; and flip angle $90^{\circ}$. Additionally, a separate T2-weighted (i.e., $b$-value $=0$ ) volume was acquired before acquisition of the diffusion-weighted volumes. A number of preprocessing steps for anatomical MRIs and diffusion tensor imaging (DTI) were performed using FSL (FMRIB's Software Library; http://www.fmrib.ox.ac.uk/fsl). For anatomical MRIs, intraacquisition head movement was estimated using affine transformation matrices resulting from eddy current correction (described below). Skull and other non-cerebral material were stripped from the T1- weighted volume and the skull-stripped brain was subject to non-linear normalization to the MNI FMRIB58_1 mm.

For DTI, eddy current correction followed by estimation of a fractional anisotropy (FA) image for each participant was achieved using v2.0, the diffusion toolkit within FSL. The eddy current correction step minimized distortions induced by eddy currents and aligned each diffusion-weighted volume to the first non-diffusion-weighted volume to correct for simple intra-acquisition head movement. Rotations applied to the diffusion-weighted volumes were also applied to the corresponding gradient directions [14]. FA was estimated using weighted linear least squares fitted to the log-transformed data. A custom FA template was constructed in MNI152 space using an iterative registration scheme. For the initial iteration, the skull-stripped FA images were registered using FLIRT, v5.5, with 12 degrees of freedom [15], to the standard FA brain provided by FMRIB (1 mm isotropic).

\section{DTI fiber tractography}

Any voxel with fractional anisotropy (FA) exceeding a predefined threshold (0.3) was classified as white matter. For each voxel classified as white matter, a streamline was initialized from the two opposing directions of the principal eigenvector. Each streamline was propagated using the fiber assignment by a continuous tracking (FACT) algorithm [16]. Propagation was terminated either if a minimum angle threshold of $50^{\circ}$ was violated or if a voxel was encountered with FA below 0.2. At each increment, the direction of propagation was parallel to the orientation of the eigenvector closest to the current streamline endpoint. The two opposing streamlines initialized from each voxel were then joined at their point of initialization to yield a single streamline. Each streamline was normalized to MNI space using the nonlinear warp computed during the preprocessing stage. This resulted in a wholebrain tractographic map in MNI space for each subject. Tractographic maps were viewed with TrackVis software [17].

Numbers of whole brain fibers, interhemispheric-connected fibers and fibers within the left and right hemisphere were counted for the KS-DCC patient and controls. Fiber tract connectivity was modeled as a network, or graph, comprising 116 nodes. The anatomical name by which each gray-matter node was labeled was taken directly from the automated anatomical labeling (AAL) [18]. Each node encapsulated a distinct gray matter region, and pairs of nodes were joined by a link if they were interconnected via a sufficient number of streamlines. Connectivity matrices were populated and binarized where matrix element quantified the connectivity between node pairs. The rows/ columns of the connectivity matrix and the binarized connectivity matrix were ordered as shown in Figure 2A, indicating all left hemisphere nodes occupied the first 58 rows/columns. Therefore, the two strongly interconnected sub-blocks along the matrix diagonal exclusively involved intrahemispheric connections, whereas the two off-diagonal sub-blocks involved inter-hemispheric connections. The matrix of the patient and mean matrices for five controls are shown in Figure 2A. The number of fibers in regions of interest were counted from the connectivity matrices and compared between controls and the patient.

\section{Results}

\section{Olfaction, taste, IQ and mirror movement assessments}

A summary of the KS-DCC patient and control characteristics is shown in Table 1. Olfactory detection and recognition scores were normal in controls as determined by criteria described in a prior study [11], whereas the patient showed impairment of both odor detection 
Control

A

B

$\mathrm{C}$
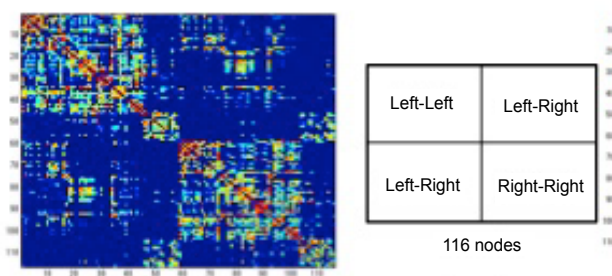

116 nodes
KAL-DCC
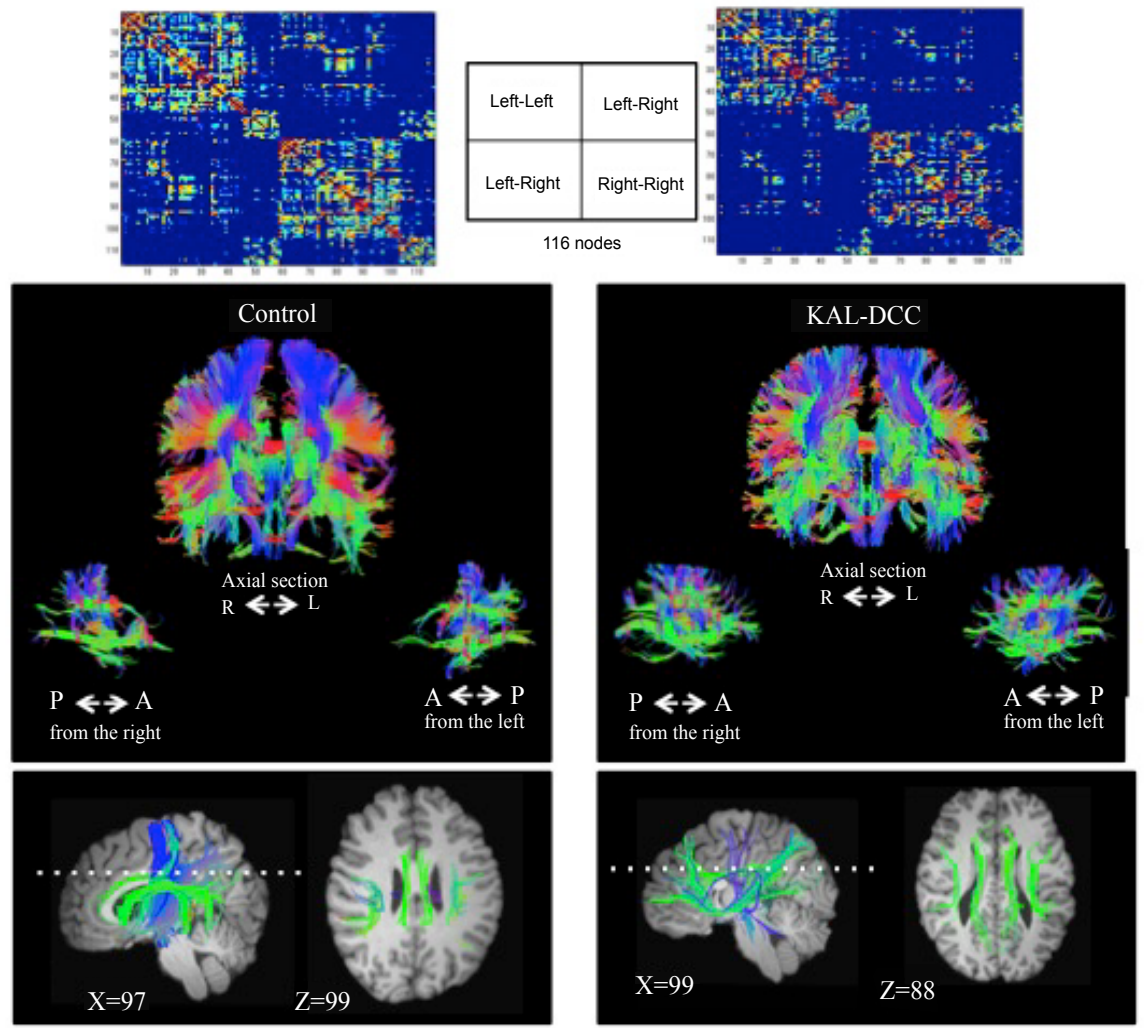

Figure 2: A: The anatomical names for labeling nodes were taken directly from automated anatomical labeling (AAL). Connectivity matrices (inter connecting streamlines) between 116 node pairs in controls and the KS-DCC patient. The matrix for controls represents the mean group value. The rows/columns of the connectivity matrices were ordered such that all left hemisphere nodes occupied the first 58 rows/columns and the right hemisphere and inter-hemispheric nodes occupied the second 58 rows/columns.

B: Streamlines inter and intra-connecting all nodes pairs in axial sections (top) and intra-connecting pairs in sagittal sections (bottom).

C: The cingulate bundle in sagittal and horizontal view in normal controls (left) and Probst bundle in sagittal and horizontal views in KS (right), A, Anterior; P, Posterior;

R, Right; L, Left.

and recognition. Five basic taste sensations were in the normal range in both the patient and controls. Neuropsychological assessment showed that WAIS FSIQ was within the normal range in both controls and the KS-DCC patient.

Assessment of mirror movements indicated that strong and high amplitude in the voluntarily moving hand was observed in all runs in both controls and the KS-DCC patient. No EMG activity was observed in the resting hand in either controls or the patient.

\section{Whole brain fibers divided into intra-hemisphere and inter- hemispheres}

Figure 2A shows connectivity matrices (inter connecting streamlines) between 116 node pairs in controls and the KS-DCC patient. The matrix for controls represents the mean group value. The rows/columns of the connectivity matrices were ordered such that all left hemisphere nodes occupied the first 58 rows/columns and the right hemisphere and inter-hemispheric nodes occupied the second 58 rows/columns. As observed in both matrices, there were fewer inter-hemispheric connections in the patient with KS-DCC compared to controls, but more intra-hemispheric connections: Streamlines inter- and intra-connecting all node pairs in axial views and intraconnecting pairs in sagittal views in control and KAL-DCC are shown in Figure 2B.

\begin{tabular}{|l|c|c|}
\hline & Controls & Kall-DCC \\
\hline Total & $859682 \pm 132676$ & 1043950 \\
\hline Right hemisphere & $381874 \pm 56295$ & 498294 \\
\hline Left hemisphere & $377119 \pm 55950$ & 488052 \\
\hline Inter-hemispheric & $100689 \pm 27857$ & 57604 \\
\hline
\end{tabular}

Table 2: The number of fibers intra-hemisphere connections and inter-hemisphere connections.

(i) Intra-hemispheric connections - The number of fibers counted within the left and right hemispheres, and those connected interhemispherically are shown in Table 2 . The total number of fibers in all brain regions from controls was $859,682 \pm 132,676$ compared with $1,043,950$ in the patient. The number of fibers in the left hemisphere was $377,119 \pm 55,950$ in controls and 488,052 in the KS-DCC patient, and in the right hemisphere, there were $381,874 \pm 56,295$ in controls and 498,294 in the KS-DCC patient. Thus, there were higher numbers of fibers in the total brain, and intra- hemispheres (bilaterally) in the KS-DCC patient than in the controls. This difference in intrahemisphere fiber numbers between controls and the patient was observed in the visualized fiber tracts (Figure $2 \mathrm{~B}$ ). In the patient, fibers from the hemispheres failed to cross the midline and formed a thick bundle intra-hemispherically running from the posterior to anterior sections. This thick bundle is termed the Probst bundle (PB) [19] had 
fibers from parietal-occipital regions and was located medially to the lateral ventricle (Figure $2 \mathrm{C}$ right). Figure $2 \mathrm{C}$ left indicates the cingulate bundle of normal controls.

(ii) Inter-hemispheric connections- In contrast, the number of fibers connecting left and right hemispheres in the KS-DCC patient was $42.8 \%$ lower compared with controls (controls: 100,689 $\pm 27,857$ and KS-DCC: 57,604). The normal CC connects the two cerebral hemispheres with a large number of fibers, as observed in the control subjects. Compared with controls, a smaller number of fibers connected the left and right hemisphere through two genua remnants in the KSDCC patient (Figure 3). We observed the presence of a sigmoid-shaped pathway, which is called sigmoid bundle, connecting the right frontal lobe with the left pareto-occipital regions (Figure 3 right: white arrow).

\section{Olfactory-related fibers in KS-DCC}

We extracted areas related to olfaction according to previous studies [20-25]. Fiber counts between these olfactory regions are shown in Table 3.

The olfactory system defined by AAL includes the olfactory tract, olfactory tubercle and anterior commissure. Since the olfactory tract is absent in this patient, we use the term "olfactory areas" to describe the olfactory tubercle and anterior commissure. The olfactory area, entorhinal cortex and rectus had inter-hemispheric fibers (indicated as "inter-hemispheric" in Table 3) and other olfactory areas such as amygdala (AMG), inferior frontal/orbitofrontal (Frontal_inf_OFC) and superior frontal/orbitofrontal (Frontal_sup_OFC) had ipsilateral fiber connections (indicated as "Right hemisphere" and "Left hemisphere" in Table 3). There were fewer inter-hemispheric fibers between olfactory-R and $-\mathrm{L}$ in the KS-DCC patient (57 in KS-DCC and $220 \pm 156$ in controls). However, other intra- hemispheric fibers (e.g. connecting between olfactory area, AMG, rectus, frontal inf. OFC and frontal sup. OFC and other inter-hemispheric fibers connecting regions such as ENT and rectus) in the patient did not differ compared with normal controls.

\section{Fibers related to motor execution}

Since the patient with KS-DCC appeared to have more intrahemispheric fibers and fewer inter-hemispheric fibers, next we determined the number of fibers connecting the AAL defined anatomical areas in KS-DCC compared with controls. We subtracted the matrix of controls (indicated in Figure 2A) from the matrix of KSDCC to examine which intra-hemispheric fibers/inter-hemispheric fibers in the patient were larger/less than those of controls. Larger numbers of fibers were observed between the left thalamus and left supplementary motor areas (SMA) and between the right thalamus and right SMA (indicated as bold in Table 4: upper panel) in the patient compared to controls (left, KS-DCC, 14,380, Controls, 1,854 \pm 796 ; right, KS-DCC, 8,731, Controls, 1,397 \pm 669 ; Figure 4A). The area of significantly reduced fibers was between the left SMA and right SMA (indicated as bold in Table 3: bottom panel; KS-DCC, 458, Controls, $10,755 \pm 4002$, i.e., $95.7 \%$ reduction; Figure 4B). Connecting fibers between the thalamus and other motor related areas (para-central and pre-central) are also shown in Table 4 . There was no marked difference in fibers connecting between the thalamus and para-central, between the thalamus and pre-central, between the SMA and para-central,
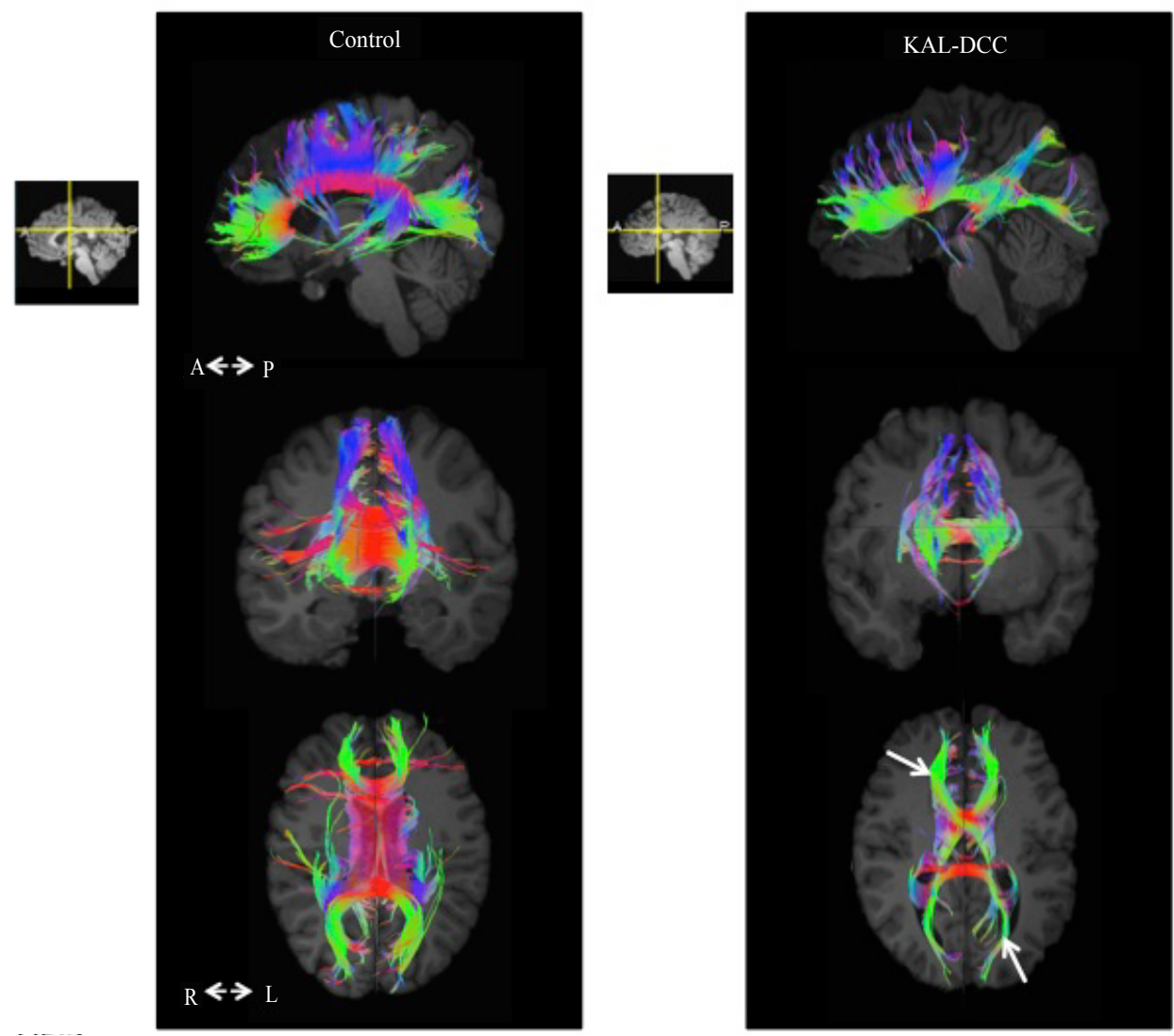

Figure 3: Inter-hemisphere connections from sagittal, coronal and horizontal (from bottom) in control and KS-DCC, R, Right; L, Left. 
Citation: Masaoka Y, Zalesky A, Tatsuno M, Okamoto S, Yoshida M, et al. (2016) Inter- and Intra-Hemispheric Fibers of Olfactory and Motor Areas in Kallmann Syndrome with Defective Corpus Callosum. J Neurol Neurophysiol 7: 380. doi:10.4172/2155-9562.1000380

\begin{tabular}{|c|c|c|c|}
\hline \multicolumn{4}{|c|}{ Right hemisphere } \\
\hline \multicolumn{2}{|c|}{ Regions } & \multicolumn{2}{|c|}{ Number of fibers } \\
\hline From & To & Controls & Kall-DCC \\
\hline \multirow[t]{4}{*}{ Olfactory } & AMG & $136 \pm 100$ & 147 \\
\hline & Rectus & $427 \pm 132$ & 603 \\
\hline & Frontal inf. OFC & $50 \pm 32$ & 39 \\
\hline & Frontal sup. OFC & $149 \pm 94$ & 63 \\
\hline \multirow{2}{*}{ AMG } & ENT & $707 \pm 333$ & 1784 \\
\hline & Frontal inf. OFC & $21 \pm 9.3$ & 0 \\
\hline \multirow{2}{*}{ Rectus } & Frontal inf. OFC & $202 \pm 98$ & 520 \\
\hline & Frontal sup. OFC & $515 \pm 311$ & 518 \\
\hline Frontal sup. OFC & Frontal inf. OFC & $826 \pm 420$ & 1020 \\
\hline \multicolumn{4}{|c|}{ Left hemisphere } \\
\hline \multicolumn{2}{|c|}{ Regions } & \multicolumn{2}{|c|}{ Number of fibers } \\
\hline From & To & Controls & Kall-DCC \\
\hline \multirow[t]{4}{*}{ Olfactory } & AMG & $81 \pm 21$ & 26 \\
\hline & Rectus & $458 \pm 221$ & 749 \\
\hline & Frontal inf. OFC & $89 \pm 65$ & 191 \\
\hline & Frontal sup. OFC & $55 \pm 20$ & 199 \\
\hline \multirow{2}{*}{ AMG } & ENT & $788 \pm 263$ & 1692 \\
\hline & Frontal inf. OFC & $75 \pm 45$ & 5 \\
\hline \multirow{2}{*}{ Rectus } & Frontal inf. OFC & $206 \pm 106$ & 572 \\
\hline & Frontal sup. OFC & $910 \pm 448$ & 485 \\
\hline Frontal sup. OFC & Frontal inf. OFC & $956 \pm 538$ & 1083 \\
\hline \multicolumn{4}{|c|}{ Inter-hemispheric } \\
\hline \multicolumn{2}{|c|}{ Regions } & \multicolumn{2}{|c|}{ Number of fibers } \\
\hline From & To & Controls & Kall-DCC \\
\hline Olfactory-R & Olfactory-L & $220 \pm 156$ & 57 \\
\hline ENT-R & ENT-L & $371 \pm 107$ & 284 \\
\hline Rectus- $R$ & Rectus-L & $432 \pm 103$ & 536 \\
\hline
\end{tabular}

Table 3: The number of fibers connected between each olfactory-related area. between the SMA and pre-central and between the pre-central and para-central within the right and left hemispheres.

\section{Discussion}

\begin{tabular}{|l|c|c|c|}
\hline \multicolumn{4}{|c|}{ Right hemisphere } \\
\hline \multicolumn{2}{|c|}{ Regions } & \multicolumn{2}{c|}{ Number of fibers } \\
\hline \multirow{2}{*}{ From } & To & Controls & Kall-DCC \\
\hline \multirow{2}{*}{ Thalamus } & SMA & $1397 \pm 669$ & 8731 \\
\hline \multirow{2}{*}{ SMA } & Para-central & $496 \pm 252$ & 344 \\
\cline { 2 - 4 } & Pre-central & $3119 \pm 828$ & 3237 \\
\hline Pre-central & Para-central & $1108 \pm 450$ & 853 \\
\cline { 2 - 4 } & Pre-central & $350 \pm 128$ & 148 \\
\hline
\end{tabular}

\begin{tabular}{|l|l|c|r|}
\hline \multicolumn{3}{|c|}{ Left hemisphere } \\
\hline \multicolumn{2}{|c|}{ Regions } & \multicolumn{2}{c|}{ Number of fibers } \\
\hline \multicolumn{1}{|c|}{ To } & Controls & \multicolumn{1}{c|}{ Kall-DCC } \\
\hline \multirow{2}{*}{ Thalamus } & SMA & $1854 \pm 796$ & 14380 \\
\hline \multirow{2}{*}{ SMA } & Para-central & $4981 \pm 955$ & 5137 \\
\cline { 2 - 4 } & Pre-central & $3349 \pm 3260$ & 2000 \\
\hline Pre-central & Para-central & $1708 \pm 544$ & 0 \\
\cline { 2 - 4 } & Pre-central & $98 \pm 55$ & 371 \\
\hline
\end{tabular}

\begin{tabular}{|l|c|c|c|}
\hline \multicolumn{4}{|c|}{ Inter-hemispheric } \\
\hline \multicolumn{2}{|c|}{ Regions } & \multicolumn{2}{c|}{ Number of fibers } \\
\hline From & To & Controls & Kall-DCC \\
\hline SMA-R & SMA-L & $10755 \pm 4002$ & 458 \\
\hline Para-central-R & Para-central-L & $1347 \pm 773$ & 27 \\
\hline SMA-R & Para-central-L & $1421 \pm 658$ & 243 \\
\hline SMA-L & Para-central-R & $202 \pm 222$ & 6 \\
\hline
\end{tabular}

Table 4: The number of fibers connected between each motor-related area.

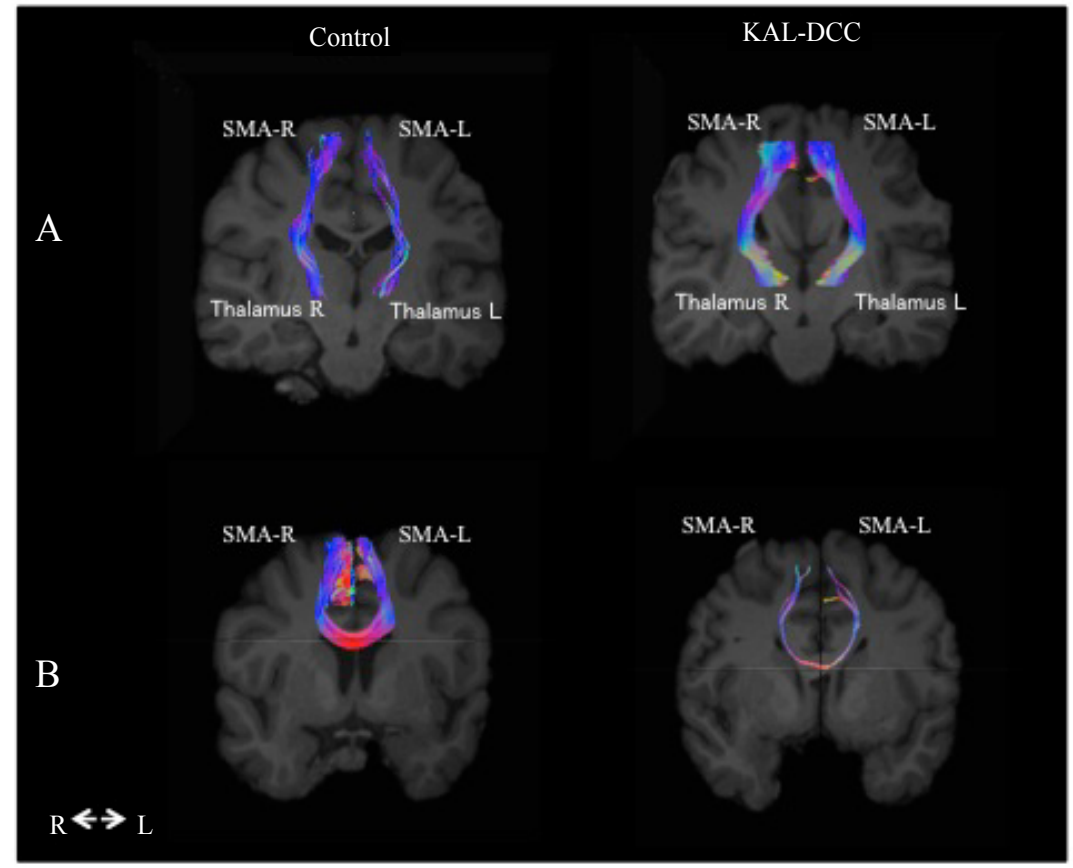

Figure 4A: Intra-hemispheric fibers connecting between left thalamus and left SMA, right thalamus and right SMA in controls (left) and the patient (right) B: Inter-hemispheric connections between right SMA and left SMA-right SMA in controls left) and the patient (right). 
In this study, we examined both inter- and intra-hemispheric white-matter connectivity in a patient with KS-DCC compared with normal healthy subjects. Overall, in KS-DCC, we identified fewer interhemispheric connections but more intra-hemispheric connections. Further, while KS-DCC had impaired odor detection and recognition, olfactory related areas revealed normal intra-hemispheric fibers. However, KS-DCC had fewer inter-hemispheric fibers between right and left olfactory areas (including olfactory tubercle and anterior commissure). We also found that the smallest numbers of fibers connecting inter-hemispheric areas were those fibers connecting left and right SMA; while the largest fiber numbers were the intrahemispheric connections between the left thalamus and left SMA and between the right thalamus and right SMA.

\section{More intra-hemispheric fibers and fewer inter-hemispheric fibers}

CC is the major commissural fiber bundle in the human brain containing about 200 million axons that are highly organized for connecting both hemispheres. Cognitive and motor functions coordinate and transfer information between the left and right brain. CC agenesis can appear as two types: complete agenesis of CC or DCC. Complete agenesis of CC causes a number of syndromes including neuropsychiatric problems [26], learning difficulties [27], sleeping disorders [28] and deficits of motor control such as mirror movements [29-31]. DCC agenesis was first described by Reil [32] who showed that the PB longitudinal bundle of fibers was aberrant. These patients lead a normal life without cognitive impairment, as the $\mathrm{PB}$ has a compensatory CC function. In this KS patient, the PB contained $\mathrm{U}$-shaped longitudinal fibers in the medial sector of the frontal regions and long fibers from occipital regions to the frontal areas that may indicate a role for plastic rewiring of CC connections.

In terms of whole brain fibers, the KS-DCC patient had higher numbers of intra-hemispheric fibers within each of the right and left hemispheres and fewer inter-hemispheric fibers. Higher numbers of fibers connected the right thalamus and right SMA and the left thalamus and left SMA in the KS-DCC patient compared with controls. Because of the absence of $\mathrm{CC}$, which serves as a massive commissural pathway, the left and right oriented cortical axons intermingled with corticospinal and thalamocortical pathways. This phenomenon was supported by a study showing a higher fractional anisotropy value of somatosensory and motor pathways in patients with DCC [26].

In contrast, the fewest number of fibers observed in the KS-DCC patient were those connecting the right and left SMA. This indicates that numbers of fibers connecting the left and right hemispheres were diminished by the absence of an intact a CC. This may explain the lack of mirror movements as discussed below.

\section{Fibers connecting olfactory related regions}

The Kal-1 gene encodes a protein, anosmin-1, which is responsible for the symptoms, observed in KS syndrome [3]. Anosmin-1 influences the development of the olfactory pathway, the olfactory bulb, olfactory tract and projections of the mitral cell to the Pir [33]. Our interest was to investigate whether the disturbance of olfactory axons in the olfactory bulb and olfactory tract would influence other fibers connecting olfactory related regions.

We found that the KS-DCC patient had normal ipsilateral connecting fibers from the olfactory tubercle to the higher order olfactory-related areas. This finding suggests that the development of fibers from the olfactory tubercle to higher olfactory regions may be independent from the development of the olfactory bulb and tract, and is not influenced by the defective axonal branching caused by anosmin-1 deficiency.

In addition, fibers between the inter-hemispheric olfactory tubercle were connected through the anterior commissure. The anterior commissure forms part of the $\mathrm{CC}$ and its olfactory perception role is to connect the right olfactory nucleus to the left olfactory tract and bulb and vice versa. There is evidence to suggest that the role of the anterior commissure in olfaction is identification of odors via memory retrieval [34]. Olfactory tubercles form part of the ventral pallidum and the striatum which have a role in cognition, emotion and reward behavior [35].

It is reasonable to assume that the patient likely had similar structural fibers as controls because these olfactory related areas such as AMG, ENT, rectus and superior/inferior OFC overlapped with areas involved in emotions [23], memory retrieval [36], guidance of emotional behavior and identifying emotions [24]. The patient had normal emotional reactions; therefore, structural connections related to emotional functions overlapped with olfaction-related regions may be normal in the patient. Therefore, olfactory deficit in this patient may only due to absence of olfactory bulb that is the first site of processing olfactory information and relays the information to subcortical and cortical areas.

In this study, only white matter connectivity was examined. Therefore, there is a need for future assessment of gray matter volume and thickness. Frasnell et al. [37], for example, reported that congenital anosmia patients exhibited a thicker primary olfactory cortex such as ENT and Pir, and also had thicker OFC compared with controls. This study suggested that congenital anosmia, like other sensory systems such as congenital vision and auditory loss, may be attributed to reduced or absent synaptic pruning caused by missing peripheral sensory inputs [37].

The relationship between cortical thickness of the olfactory areas and fibers of the white matter surrounding these areas is unknown, and requires further investigation in patients who suffer from congenital or non-congenital anosmia.

\section{Absence of mirror movement}

The KS-DCC patient had no mirror movements, which is often observed in X-linked Kall syndrome [13,29,31,38]. Interestingly, the patient in this study had KS syndrome as well as DCC. Complete agenesis of the CC also causes a deficit of motor control, including mirror movements [39-41]. Normally, mirror movements occur due to activity in the contralateral motor area when there is a lack of interhemispheric inhibition from the primary motor cortex associated with an intended voluntary movement. Thus, inter-hemispheric inhibition between the left and right primary motor cortices is mediated by the CC. This means that effective inter-hemispheric inhibition is necessary for correct motion and for the suppression of mirror movements. However, inter-hemispheric connectivity would also be needed in order to produce mirror movements - so, in this KS patient we speculate that an absence of mirror movements results from significantly reduced interhemisphere connectivity. This may lead to a failure of transmission of a mirror signal; alternatively, lack of mirror movements may be due to a failure of inhibition across the hemispheres.

\section{Limitation and Further Research}

In this study, whole brain-tractography was performed to examine cortico-cortical connectivity. Although this approach is more sensitive to network disruptions, the results are contingent on the accuracy of 
the tractography algorithm. FACT streamline tracking algorithm was used in this study. The FACT algorithm is simple, computationally inexpensive, and has been demonstrated to yield robust tractographic maps. However, streamline tracking algorithms in general are known to have difficulty in following long distance fibers, either due to partial volume effects, a poor fit of the diffusion tensor, or simply due to noise [6]. In addition, given we only examined one patient, we were not able to test for associations between olfaction and motor deficits statistically, therefore, one patient might adequately characterize patients with KS and agenesis of CC. The findings reported in this study might potentially be interpreted as effects arising from the absence of cognitive and motor deficits in this patient.

DTI tractography has been tested in patients with KS, mainly for the presence and mechanisms of mirror movement [13,38] and in patients with agenesis of CC and DCC [29,31] to investigate cognitive and emotional functions as well as mirror movement. These studies on KS and CC or DCC agenesis have been investigated separately. Here, we tested for the first time a rare patient with KS-DCC.

We are also interesting to know a relationship between gray matter volume and numbers of fibers connecting among gray matter [39] in the KS-DCC. A similar study including measurement of gray matters with a larger population, more strictly defined patient population (KS with/without agenesis of CC or DCC and define complete genetic characterization) may offer further insights into the neurological profile of KS. Our study is limited to genetic information for the patient, however, it is possible to assume that that Kal-1, which encodes anosmin-1 might cause the abnormal development of CC. Agenesis of CC has been suggested to accompany KS [13,31] because anosmim-1 is present in oligodendroglial cells in the CC and the pyramidal tract during development [3]. Thus, olfactory-brain development guided with anosmin-1 could have a key role in determining the midline and the subsequent development of the brain.

\section{Acknowledgement}

Yuri Masaoka was supported by Showa University grant-in-aid for research project. This work was supported by the Australian National Health and Medical Research Council (Career Development Fellowship (ID: GNT1047648) to Dr Zalesky. Christos Pantelis was supported by an Australian NHMRC Senior Principal Research Fellowship (ID: 628386). We gratefully thank Dr. Katsutoshi Murata (Siemens, Japan) for technical support with MRI acquisition and Dr. Thorsten Feiweier (Siemens Healthcare, Germany) for providing the diffusion sequence prototype used in this study.

\section{References}

1. Seminara S, Hayes F, Crowley W (1998) Gonadoropin-releasing hormone deficiency in the human (idiopathic hypogonadotropic hypogonadism and Kallmann's syndrome): pathophysiological and genetic consideration. Endocr Rev 19: 521-539.

2. Hardelin JP (2001) Kallmann syndrome: towards molecular pathogenesis. Mol Cell Endocrinol 179: 75-81.

3. Clemente D, Esteban PF, Del Valle I, Bribián A, Soussi-Yanicostas N, et al (2008) Expression pattern of Anosmin-1 during pre- and postnatal rat brain development. Dev Dyn 237: 2518-2528.

4. Whitlock KE (2005) Origin and development of GnRH neurons. Trends Endocrinol Metab 16: 145-151.

5. Pierpaoli C, Jezzard P, Basser PJ, Barnett A, Di Chiro G (1996) Diffusion tensor MR imaging of the human brain. Radiology 201: 637-648.

6. Zalesky A, Fornito A (2009) A DTI-derived measure of cortico-cortical connectivity. IEEE Trans Med Imaging 28: 1023-1036.

7. Zalesky A, Fornito A, Harding IH, Cocchi L, Yücel M, et al. (2010) Whole-brain anatomical networks: Does the choice of nodes matter? Neuroimage 50: 970983.
8. Oldfield RC (1971) The assessment and analysis of handedness: The Edinburgh inventory. Neuropsychologia 9: 97-113.

9. Rombaux P, Duprez T, Hummel T (2009) Olfactory bulb volume in the clinical assessment of olfactory dysfunction. Rhinology 47: 3-9.

10. Wainwright M, Wright MJ, Geffen GM, Geffen LB, Luciano M, et al. (2004) Genetic and environmental sources of covariance between reading tests used in neuropsychological assessment and IQ subtests. Behav Genet 34: 365-376.

11. Masaoka Y, Pantelis C, Phillips A, Kawamura M, Mimura M, et al. (2013) Markers of brain illness may be hidden in your olfactory ability: a Japanese perspective. Neurosci Lett 549: 182-185.

12. Ogawa H, Wakita M, Hasegawa K, Kobayakawa T, Sakai N, et al. (2005) Functional MRI detection of activation in the primary gustatory cortices in humans. Chem Senses 30: 583-592.

13. Mayston MJ, Harrison LM, Quinton R, Stephens A, Krams M, et al. (1997) Mirror movements in x-linked Kallmann's syndrome. Brain 120: 1199-1216.

14. Leemans A, Jones DK (2009) The B-matrix must be rotated when correcting for subject motion in DTI data. Magn Reson Med 61: 1336-1349.

15. Jenkinson M, Smith S (2001) A global optimisation method for robust affine registration of brain images. Med Image Anal 5: 143-156.

16. Mori S, Crain BJ, Chacko VP, van Zijl PC (1999) Three-dimensional tracking of axonal projections in the brain by magnetic resonance imaging. Ann Neurol 45: $265-269$

17. Wedeen VJ, Wang RP, Schmahmann JD, Benner T, Tseng WY, et al. (2008) Diffusion spectrum magnetic resonance imaging (DSI) tractgraphy of crossing fibers. Neuroimage 15: 1267-1277.

18. Tzourio-Mazoyer N, Landeau B, Papathanassiou D, Crivello F, Etard O, et al. (2002) Automated anatomical labeling of activations in SPM using a macroscopic anatomical parcellation of the MNI MRI single-subject brain. Neuroimage 15: 273-89.

19. Probst M (1903) Ueber den Bau des balkenlosen Grosshirns, sowie ube Mikrogirie und Heterotopie der grauen substanz. Arch Psychiatr Nevenkr 34: 709-786.

20. Bensafi M, Rouby C (2007) Individual differences in odor imaging ability reflect differences in olfactory and emotional perception. Chem Senses 32: 237-244.

21. Gottfried JA, Deichmann R, Winston JS, Dolan RJ (2002) Functional heterogeneity in human olfactory cortex: An event-related functional magnetic resonance imaging study. J Neurosci 22:10819-10828.

22. Masaoka Y, Koiwa N, Homma I (2005) Inspiratory phase-locked alpha oscillation in human olfaction: Source generators estimated by a dipole tracing method. J Physiol 566: 979-997.

23. Rolls ET, Kringelbach ML, de Araujo IE (2003) Different representations of pleasant and unpleasant odors in the human brain. Eur J Neurosci 18: 695-703.

24. Royet JP, Plailly J, Delon-Martin C, Kareken DA, Segebarth C (2003) fMR of emotional responses to odors: influence of hedonic valence and judgment, handedness and gender. Neuroimage 20: 713-728.

25. Sobel N, Prabhakaran V, Zhao Z, Desmond JE, Glover GH, et al. (2000) Time course of odorant-induced activation in the human primary olfactory cortex. $J$ Neurophysiol 83: 537-551.

26. Kasprian G, Brugger PC, Schöpf V, Mitter C, Weber M, et al. (2013) Assessing prenatal white matter connectivity in commissural agenesis. Brain 136: 168179

27. Párraga HC, Párraga MI, Jensen AR (2003) Cognitive, behavioral, and psychiatric symptoms in two children with agenesis of the corpus callosum: Case report. Int J Psychiatry Med 33: 107-113.

28. Nielsen T, Montplaisir J, Lassonde M (1992) Sleep architecture in agenesis of the corpus callosum: Laboratory assessment of four cases. J Sleep Res 1 : 197-200.

29. Beaulé $V$, Tremblay S, Théoret $H$ (2012) Interhemispheric control of unilateral movement. Neural Plast 2012: 627816.

30. Tovar-Moll F, Moll J, de Oliveira-Souza R, Bramati I, Andreiuolo PA, et al. (2007) Neuroplasticity in human callosal dysgenesis: A diffusion tensor imaging study. Cereb Cortex 17: 531-541.

31. Peng J, Charron $F$ (2013) Lateralization of motor control in the human nervous system: genetics of mirror movements. Curr Opin Neurobiol 23: 109-118. 
Citation: Masaoka Y, Zalesky A, Tatsuno M, Okamoto S, Yoshida M, et al. (2016) Inter- and Intra-Hemispheric Fibers of Olfactory and Motor Areas in Kallmann Syndrome with Defective Corpus Callosum. J Neurol Neurophysiol 7: 380. doi:10.4172/2155-9562.1000380

32. Reil LC (1812) Mangel des mittleren und freyen Theils des Balkens im Menschengehirn. Arch Physiol 11: 314-344.

33. Soussi-Yanicostas N, de Castro F, Julliard AK, Perfettini I, Chédotal A, et al. (2002) Anosmin-1, defective in the X-linked form of Kallmann syndrome, promotes axonal branch formation from olfactory bulb output neurons. Cell 109: 217-28.

34. Cattarelli M (1982) The role of the medial olfactory pathways in olfaction: Behavioral and electrophysiological data. Behav Brain Res 6: 339-364.

35. Heimer L, Zahm DS, Alheid GF (1995) Basal Ganglia: The rat nervous system. (2ndedn), 2. Academic Press, San Diego.
36. Masaoka Y, Sugiyama H, Katayama A, Kashiwagi M, Homma I (2012) Remembering the past with slow breathing associated with activity in the parahippocampus and amygdala. Neurosci Lett 521: 98-103.

37. Frasnelli J, Fark T, Lehmann J, Gerber J, Hummel T (2013) Brain structure is changed in congenital anosmia. Neuroimage 83: 1074-1080.

38. Koenigkam-Santos M, Santos AC, Borduqui T, Versiani BR, Hallak JE, et al. (2008) Whole-brain voxel-based morphometry in Kallmann syndrome associated with mirror movements. AJNR Am J Neuroradiol 29: 1799-1804.

39. Manara R, Salvalaggio A, Favaro A, Palumbo V, Citton V, et al. (2014) Brain changes in Kallmann syndrome. AJNR Am J Neuroradiol 35: 1700-1706. 